\title{
Antropología «compleja» de las emociones humanas
}

\author{
EUGENIA RAMÍREZ GOICOECHEA \\ UNED, Madrid
}

Resumen. Hablar, escribir y pensar en las emociones está de moda. Nosotros nos sumamos a la corriente porque pensamos (y sentimos) que ya era hora. Desde una perspectiva interdisciplinar abordamos la complejidad concentrándonos en cuatro temas básicos, algunos ya clásicos: la universalidad de las emociones y la unidad psíquica del hombre/mujer, el debate entre cognición y emoción, los efectos de las emociones en lo social y de lo social en las emociones y, por fin, la propuesta de una teoría experiencial compleja de la emoción. Con esto no pretendemos agotar, ni mucho menos, el debate, sino sólo sugerir y orientar la mirada para futuros/as exploradores/as en este tema tan fascinante.

\section{Introducción}

El interés académico y popular por las emociones está en pleno auge. Desde que nos cuentan la multitud de inteligencias que pueden describirse del ser humano, incluyendo la emocional (Gardner, 1983) hasta la explosión bibliográfica y massmediática sobre la vida afectiva, el tema no ha dejado de ocupar un espacio creciente en el discurso de la «postmodernidad».
ABSTRACT. It has become fashionable to talk and write about the human emotions. We do think that it is time we social (and other) scientists pay more attention to the emotionality of (wo)men. Our approach is an interdisciplinary one, since we believe it is the best way to deal with the complexity of this phenomena. We will concentrate on four major aspects of it: the universality of emotions, the discussion on the relationships between emotion and cognition, the social effects of emotions and the emotional effects of the social, and, at last, the embodied nature of emotion. Ours is an open-ended «story», and we hope to contribute to the discussion and encourage further explorations on such a fascinating topic.

S. J. Williams cita los siguientes vectores sociológicos y paradigmáticos para explicar la «mayoría de edad» de las emociones en la preocupación del quehacer de sociólogos, antropólogos, psicólogos, politólogos. Después de rastrear la permanencia oculta de la cuestión de las emociones en los fundadores de la sociología moderna (Durkheim, Weber, Marx, Simmel), nos pone al día de la actualidad del estudio de las emociones en la sociología y antro- 
pología de la década de los ochenta. Concluye que este resurgimiento del interés por lo afectivo y emotivo se debe principalmente a los siguientes factores: a) $\mathrm{La}$ crítica a la filosofía y sociología racionalista y su concepción del hombre/mujer; b) La reintroducción de lo biológico en la explicación social y la importancia del cuerpo en la constitución del sujeto; c) La comercialización y comodificación de lo emocional por parte de la industria del consumo, del ocio y los mass media; $d$ ) La expansión corporativa profesional sobre el ámbito del self, sus avatares, manejo y vicisitudes, como un campo de práctica terapéutica, y e) La incorporación de lo afectivo-emocional en la agenda política, como campo de derechos y libertades en distintos contextos «glocales» (Williams, 2001:1-16). Lo que está claro es que más que como artefactos, las emociones deberían ser entendidas como procesos complejos que involucran muchas dimensiones del ser social del humano.

Nosotros no podemos explorar todas estas cuestiones en este breve ensayo sobre tema tan complejo, diverso y complicado de definir. Nuestro propósito es introducir algunas de las claves de la discusión que los fenómenos afectivos han suscitado en las ciencias humanas, especialmente la Antropología, pero también la Sociología y la Psicología. Desde ahí proponemos explorar algunos caminos que incorporen una visión filoontogenética, pero también sociocultural de las emociones humanas, lo que implicará, necesariamente, cierta «inflación» bibliográfica. Serán más las puertas abiertas que las soluciones dadas, pero eso es parte de los límites de nuestro trabajo intelectual. Lo que se pretende es ofrecer, en un esfuerzo de coherencia y articulación discursiva, una guía (incompleta necesariamente) de la mirada, el estímulo para seguir diferentes pistas. Éstas se concentran en torno a cuatro temas o problemas principales: la universalidad de las emociones y la unidad psíquica de la especie humana, el dualismo entre cognición y emociones, la profunda dimensión social de lo afectivo y emocional y, por último, una tentativa de descripción biosocial, experiencial y encarnada del sujeto como persona, en donde lo emocional se integra como elemento básico y constituyente.

\section{La universalidad de las emociones} y la unidad psíquica de la especie humana

La afirmación de que las emociones humanas son generales a nuestra especie suele basarse en argumentos filogenéticos y neurofisiológicos. Ya Darwin expresó el significado evolutivo de las emociones (Darwin, 1965). Hay una positiva correlación filogenética entre inteligencia y emoción desde los invertebrados a los humanos. Las emociones estarían ligadas al comportamiento defensivo y al establecimiento de vínculos sociales.

El sistema límbico es cinco veces mayor en el humano que en el mono (Izard, 1979). Aunque hay evidencias de que éstos también sufren emocionalmente ante la separación súbita y prolongada de la madre (Harlow y Harlow, 1962; Reite y Short, 1981), la sonrisa y el llanto son específicamente humanos, como sistemas de expresión de las emociones, en relación a estrategias adaptativas y de supervivencia de nuestra especie (Montagu, 1959), dirigidas a reclamar el cuidado de los padres en la larga ontogenia humana (Ainsworth et al., 1974). El vínculo afectivo entre infante y cuidadores tiene una función biológica esencial que sería la de proteger al niño de los predadores (Bowlby, 1969) Según Zajonc (1984), las reacciones emocionales categorizan nuestro entorno en clases de objetos y acontecimientos en términos de peligro o seguridad. La musculatura y las habilidades para expresar emociones están presentes en todo bebé sano en el nacimiento. Estos mismos muestran capacida- 
des tempranas para detectar las expresiones faciales de sus cuidadores.

Las emociones están ligadas a procesos neuroquímicos y neurofisiológicos del sistema nervioso autónomo, entre estos últimos el cambio de la conductividad eléctrica de la piel, dilatación de la pupila, alteración del pulso y la presión sanguínea, cambios en la transpiración y respiración, secreción de distintas hormonas, etc. De ahí podríamos hablar de una «neuroquímica» de las emociones (Eibl-Eibesfeldt, 1993).

Esto justificaría el carácter común de algunas de ellas para todos los humanos, que es precisamente la orientación de los trabajos de P. Ekman y C. Izard, para quienes existen evidencias crossculturales y etológicas humanas, tanto en niños normales como en niños ciegos o sordomudos (Eibl-Eibesfeldt, 1993) de determinadas emociones «básicas» como alegría, sorpresa, tristeza, enfado y asco (Ekman, 1992; Izard y Buechler, 1980). Es a través de programas motores que dirigen los movimientos faciales, con su multitud de músculos y nervios exclusivamente humanos, como la especie humana expresa e interpreta las emociones (Ekman, 1979; 1980). Desde este enfoque, la modelización neuronal de la producción y expresión de las emociones sería innata y sólo el cuando, el cómo y el control de la intensidad de las mismas variaría culturalmente ${ }^{1}$. Por otro lado, parece haber una especialización neurológica en el reconocimiento de la posición de las caras y, por ende, en el reconocimiento de la direccionalidad de la mirada (Perret et al., 1982, 1995). El reconocimiento de la modularidad en el tono de voz así como otras expresiones corporales (posturas) completarían el espectro de posibilidades experienciales y comunicativas de las emociones.

Esta orientación generalista se fundamenta en la idea de una unidad psíquica para la especie humana, perspectiva apoyada - a pesar de sus problemas para incorporar lo emocional (cf. infra) - por la psicología evolucionista (Mithen, 1996; Barkow, Cosmides y Toolby, 1992; Donald, 1991; Sperber, 1994) por la que se justifica la universalidad de una psicología humana especializada en dominios (Gómez y Núñez, 1998) a partir de la dotación específica del homo sapiens en un entorno con determinadas regularidades medioambientales como es el Pleistoceno y que ha dado lugar a sistemas clasificatorios (Atran, 1990, 1998) y preceptuales similares entre culturas (Berlin y Kay, 1991).

La Antropología, desde sus comienzos, también se ha planteado este problema, en el contexto de su especificidad como disciplina (Stocking, 1982). La idea de la unidad psíquica del hombre/mujer se ha llevado mal con la idea de la diversidad cultural entre los grupos humanos y, sin embargo, no sin dificultades, la antropología ha mantenido los dos extremos a la hora de entender la humanidad (Shore, 1996) ${ }^{2}$.

Desde esta perspectiva universalista, las emociones generales básicas tendrían reflejo en las construcciones lingüísticas elaboradas para «denotar» este tipo de experiencia. A pesar de las diferencias fonológicas, gramaticales y semánticas de las distintas lenguas, existiría una estructura semántica subyacente común a la mayoría de ellas en cuanto a la expresión de las emociones. Los humanos usarían el mismo marco descriptivo a la hora de ubicar el significado afectivo de conceptos, incluyendo valores, estereotipos, actitudes, sentimientos. Para Osgood, May y Miron (1975) habría suficiente evidencia crosscultural para afirmar esto. Levy (1984) afirma que los términos emocionales usados entre los tahitianos como en los newar refieren posiblemente a tendencias focales universales y que la diferencia reside en los limites a los que dichos términos se aplican. En esa misma perspectiva se sitúan Moore, Romney y Hsia (1999) en su análisis crosscultural de los significados de los términos 
para emociones entre sujetos hablantes de chino, inglés y japonés. Su postura es que existen estructuras semánticas comunes para los términos utilizados para nombrar emociones. Esta participación común de significados es mayor que incluso para términos utilizados para nombrar categorías de animales (Hermann y Raybeck, 1981) o de parentesco. Una de las críticas atribuibles a este tipo de trabajos es su elevado sesgo positivista y el tratamiento descontextualizado y a-experiencial de la emoción. El carácter discreto de dichas emociones «básicas» tampoco está claro.

Wierzbicka (1986) intenta mediar en esta polémica sin desbaratar, no obstante, sus presupuestos de partida. Uno de los problemas de las teorías naturalistas es que los términos que utilizan constituyen parte de la taxonomía popular inglesa, y no constituyen un marco analítico libre de dicha cultura. Por ello propone analizar las emociones desde un metalenguaje semántico general basado en palabras clave «libres» de cultura como «decir», «querer», «desear» (want), sentir, pensar, «bueno», «malo», lexicalmente existentes en todas las culturas, diversamente hiper o hipo cognizados. Wierzbicka (1986) asume que la no lexicalización de una emoción no significa que no se encuentre en una cultura, sino que los miembros de la misma no la han encontrado suficientemente saliente. Esto daría pie a hablar de cierta especificidad de dominio para las emociones en la naturaleza humana (Wierzbicka, 1994). Pero incluso aquí podría argüirse la existencia de culturas que no distinguen entre sentir y pensar, como en los Ifaluk de Micronesia (Lutz, 1982) o entre los Sami ${ }^{3}$.

La respuesta más contundente a los excesos «universalistas» ha venido de la parte de la Psicología crosscultural y la Antropología psicológica, reubicando las emociones en el terreno de lo social y cultural. La perspectiva adoptada ha sido denominada «constructivista», frente a la «organísmica», descrita hace unos momentos.
La Psicología crosscultural tomó gran interés por el tema de las emociones, dándole un giro culturalista, nada "psicologista», relacionándolo estrechamente con la construcción de la persona y del «self» (Shweder y LeVine, 1984; Rosaldo, 1980; 1984; Levy, 1984), insistiendo en la variedad de experiencias entre culturas, y la imposibilidad de referirse a lo mismo desde un punto de vista trascultural. Para Shweder (1994), por ejemplo, las emociones no son objetos naturales («natural kinds») como otros objetos del mundo fenoménico. Ahí reside uno de los problemas conceptuales fundamentales en la discusión. Este relativismo máximo es una de las formas que adopta la orientación constructivista, que hace residir en la cultura la verdadera posibilidad de la construcción, expresión y comunicación significativas de las emociones, las cuales se constituyen en torno a vocabularios compartidos, convenciones, rituales, tal como Geertz (1973) propuso para los símbolos. El objetivo es reintegrar la vida afectiva a sus relaciones con el contexto social y cultural (Lutz, 1988), al que contribuyen, a su vez, a construir. En esa medida, las emociones mediatizan el juego social, la interacción y la contestación en el seno de otros sistemas de referencia jerárquicos más vastos (Abu-Lughod y Lutz, 1990). Desde esta visión interaccionista de lo social y del poder, puede entonces hablarse de una micropolítica de las emociones y de un cuerpo «discursivo» de éstas (Foucault, 1988). Desde esta aproximación, el urso emocional constituye parte fundamental de la teoría de las emociones de una sociedad contribuyendo a la constitución (Heelas, 1996; Solomon, 1984) y ordenación de éstas (Dunn, Brown y Beardsall, 1991; Crapanzano, 1994) mediante la narración y la recitación.

Uno de los problemas principales, sobre los que este constructivismo ha desarrollado su argumento es que se han metido en el mismo saco emociones «básicas», 
como miedo, alegría, sorpresa, etc., con otras más «complejas», como la ansiedad, el odio, el amor, el arrepentimiento, el orgullo, la culpa o la vergüenza (cf. infra) y cuyos patrones organísmicos tanto neurológicos como sensorimotores no están $\tan$ claros $^{4}$. Es obvia la diferencia entre sorprenderse cuando algo entra súbitamente en nuestro campo de la experiencia como cuando vamos caminando y nos cae algo encima, a hacerlo porque un ser querido no ha recordado nuestro aniversario. De la misma manera, no es lo mismo angustiarse por la proximidad de un vacío, a sentir lo mismo ante la inminencia de una oposición académica. No podemos asegurar que en el caso de las emociones denominadas más primarias no haya «trabajo» cultural (Lutz, 1983). De hecho, como veremos enseguida, la importancia del «otro» y de una situación interactiva en la modularidad de la emoción «primaria» también ha de tenerse en cuenta. Pero lo cierto es que en las emociones «complejas» los procesos mentales y experienciales están ligadas en mucha mayor medida a procesos de aprendizaje (Harris et al., 1989) y construcción de la relación y la identidad, por tanto, también a referentes sociales y culturales.

Dos han sido las críticas más comunes a los excesos «culturalistas». Primero, su olvido de las dimensiones corporales de lo emocional «desnaturalizándolas» del todo, $\mathrm{y}$, segundo, que la emoción no puede reducirse experimental ni subjetivamente al discurso sobre la misma (Rosenberg, 1990), a pesar de posibles propiedades estructurantes y actualizantes.

¿Cómo echar luz sobre algo que nos parece humanamente tan general pero a la vez tan particular? Vamos primero a volver sobre la crítica de la unidad psíquica de lo humano, para, a partir de ahí, contribuir humildemente a resituar el debate.

La distinción entre convención y arbitrariedad que hace B. Shore (Shore, 1996) nos parece útil para iluminar la discusión sobre la universalidad o no de las emociones. Las convenciones son creaciones humanas. Son indeterminadas hasta un límite pero no arbitrarias, inmotivadas. En algún otro lugar hemos enfatizado lo mismo sobre las posibilidades, dentro de ciertas constricciones, de la acción humana con sentido (Ramírez Goicoechea, 1991; 1998). Es decir, una emoción siempre tiene una indudable incardinación neurofisiológica, pero ésta no es ajena al sistema sociocultural en que el universo emocional se constituye y adquiere sentido para el sujeto - precisamente por su instauración social, compartida, La forma de esta constitución es relativamente impredecible, convencional, pero no es inmotivada, puesto que está determinada dentro de ciertos límites constrictivos (D'Andrade, 1992) ${ }^{5}$. En cuanto a las emociones, dichos límites vendrían por los propios requisitos de estructuración de la tarea (Bates, 1979; Gibson, 1996), entre los que se incluye lo que se puede y cómo se puede hacer con el cuerpo - tanto al nivel funcional como expresivo del cuerpo-, así como por los formatos de comprensión e interpretación ofrecidos por el marco sociocultural en términos de selección de metáforas y etnoteorías diversas ${ }^{6}$. Por otro lado, además, la unidad psíquica del humano no se refiere exclusivamente a las estructuras del cerebro y las características comunes del sistema nervioso humano en determinado momento evolutivo de la especie. Si esto tiene importancia es porque nuestra dotación neurofisiológica y biológica al nacer implica un nivel de maduración que nos hace depender extraordinariamente del entorno para sobrevivir y desarrollarnos. Desde el punto de vista neurológico, nuestro cerebro se caracteriza por su tremenda apertura y plasticidad, como un órgano abierto que se reorganiza continuamente a partir de la experiencia y de una historia de transformación continua en donde «hardware» $\mathrm{y}$ «software» son interdependientes (Edelman, 1992; Varela et al., 1991; Freeman 
y Skarda, 1987; Dreyfuss, 1979; Bruner, 1996).

Si pensamos que el cerebro y su actividad es un sistema en constante autoconstrucción, no podemos separar lo orgánico de lo que le sucede y cómo se manifiesta. Si pensamos que el cerebro sigue creciendo «estructuradamente» en los primeros años de vida ${ }^{7}$, no podemos decir que es algo dado, materia prima de cambios ulteriores. Al contrario, visto como un sistema que se desarrolla autoconstituyéndose y autoorganizándose (Edelman, 1992; Changeux, 1983; Erdi, 1988) al hilo de las primeras experiencias y reorganizándose con cada nueva experiencia (Freeman, 1991), resulta que la psyque es algo diferente -más- que la dotación neurológica de la especie. Por tanto, la unidad psíquica de la humanidad no puede referirse exclusivamente a un sustrato biológico común, abstraído de sus condiciones de desarrollo (Oyama, 1992), sino en términos del entorno y el contexto histórico/biográfico (Shore, 1996) en que éste se desarrolla y al que colabora a construir ${ }^{8}$. Tendríamos que hablar mas bien del sujeto como unidad biosocial, a pesar de sus dificultades metodológicas (Hinde, 1991). De lo que trataría sería de contemplar integradamente al hombre/mujer, donde lo biológico/psicológico/social está inextricablemente unido (Morin, 1973; Goldschmidt, 1993; Freund, 1988), siendo uno condición y resultado a la vez del otro, como da cuenta la peculiar ontogenia humana (Robertson, 1996; Toren, 1993). Se trataría de verlo como una «persona» (Ingold, 1990, 1991; Carrithers, 1985; Shweder y Levine, 1984; Toren, 1998), «un agente creativo dentro de un campo total de relaciones cuyas transformaciones describen un proceso evolutivo» (Ingold, 1991; cf. Maturana y Varela, 1992).

Por tanto, hablar de la especie humana también es hablar de su potencialidad posible ${ }^{9}$, gracias también a las «tecnologías» de la civilización en el sentido histórico social (Elías, 1982) y ontogenético (cf. infra). Como dicen Gumperz y Levinson (1991) para lo cognitivo, lo emocional tampoco puede ubicarse totalmente en el individuo, sino que es también incorporado en prácticas culturales y sistemas simbólicos (cf. Hutchins y Hazlehurst, 1995) que las soportan y promueven ${ }^{10}$. Y si para las emociones básicas se habla de correspondencias neurofisiológicas «universales», para los «estados emocionales» complejos no está claro que haya patrones faciales determinados, ni que pueda hablarse de respuestas neurológicas y corporales comunes (Averill, 1996a).

La universalidad tiene, por fin, otra lectura no exclusivamente genética o evolutiva. Desde el punto de vista comparativo, de lo que se trata, como Durkheim y Mauss (1963) sostuvieron, es de lo común en los procesos sociales humanos, en el sentido de que las propiedades de la mente sólo son posibles a partir de la experiencia social, sea ésta todo lo diversa que sea a lo largo de las diversas culturas. La cuestión es que todo proceso humano es (no «está») socialmente mediado, experimentalmente vivido, y esto es común en todas las culturas. El aspecto dialógico, sobre el que nos extenderemos en el siguiente apartado, independientemente de todos sus matices crossculturales, es constitutivo de una humanidad reconocible como tal. Desde el punto de vista cognitivo y evolutivo, los trabajos de Jerome Bruner y su escuela (Bruner, 1974; Bruner, Greenfield y Olver, 1966; Olson, 1980; Trevarthen 1980, 1986; Kaye, 1977, 1980; Butterworth, 1996) asî lo ponen de manifiesto. Este aspecto contextual y situacional matizaría las necesarias correlaciones neurofisiológicas entre experiencia y expresión de las emociones tan queridas por una aproximación generalista y universal de las emociones. Pero, además, supone una fuerte crítica a la perspectiva de una emoción inscrita en el individuo ${ }^{11}$, instancia, concreción particular de la especie. No se puede banalizar los 
aspectos comprensivos e interpretativos de las situaciones específicas en que se experimentan las emociones, y muchas de éstas se concretan en otros que también experimentan y expresan emociones (Ginsburg y Harrington, 1996) ${ }^{12}$.

\section{Cognición y emoción, racionalidad $y$ afectos}

Es de todos conocida la tradición filosófica occidental, heredera del judeocristianismo y el cartesianismo que relega lo emocional a lo visceral y lo pasional, instintos «bajos» ligados a lo corporal frente a lo mental (Shilling y Mellor, 1996). El problema reside en la dicotomía cultura-mente (Shore, 1996) y sus conexiones con otros dualismos (Varela et al., 1991; Johnson, 1987; Putnam, 1999). Desde esta perspectiva dualista, las emociones, como los sentidos, «entorpecen» la razón. Esta visión racionalizante de la modernidad es deudora de una visión «cognitivista» que sitúa al racionalismo como el logro más visible de nuestra reciente historia (occidental), dejando de lado otras lecturas, discursos y problemáticas menos publicitadas de nuestra tradición de pensamiento (Latour, 1992). Sin embargo, y muy a su pesar, la emoción ha sido una preocupación central «oculta» en el racionalismo occidental (Barbalet, 1998): cómo justificar sus efectos, cómo construir un individuo desencarnado que, sin embargo, «desgraciadamente», siente y padece.

Desde la Sociología, Émile Durkheim (1982) entendió el progreso como la evacuación de los afectos, ligados a la mentalidad primitiva. Paradójicamente, reconoció la importancia de lo emocional a la hora de constituirse la grupalidad y sus vínculos (cf. infra). Del mismo modo, Max Weber situó la emoción del lado de la irracionalidad, de la experiencia incomprensible intelectualmente y, en el fondo, como una desviación o perturbación de la racionalidad (Favret-Saada, 1994).

La Antropología no ha ido a la zaga en este sentido. Si Kluckhohn se adscribía a las teorías naturalistas dominantes de unas emociones básicas «coloreadas» culturalmente, para la escuela de Cultura y Personalidad la emocionalidad apuntaba a personalidades modales $\mathrm{y}$ formas de compromiso entre las pulsiones de la naturaleza y el control cultural (Levy, 1984). Esta dualidad mente/cuerpo ha sido sostenida también por los trabajos de Lévi-Strauss, donde las emociones, como subproductos corporales, no tienen cabida en una Antropología de la «mente» (Levy, 1984).

Este dualismo se ha querido apoyar en una relativa evidencia neurofisiológica de predominancia de uno u otro hemisferio cerebral: el izquierdo para lo cognitivo; el derecho para lo emotivo (Cacioppo y Petty, 1981; Tucker, 1981). Es cierto que hay evidencias experimentales sobre la implicación del hemisferio derecho en la expresión y reconocimiento de las emociones (Carlson, 1999) y que esta disimetría para lo emocional parece más antigua filogenéticamente hablando que para otros procesos cognitivos (Davidson, 1985). Pero lo cierto es que ambos hemisferios están mutuamente involucrados en múltiples procesos y tipos de inteligencia (Rose y Mesulam, 1979; LeDoux, 1998) y parece que las regiones cerebrales funcionan estructurando y reestructurándose constantemente permitiendo hasta cierto punto cierto «uso compartido» para distintas funciones (Calvin, 1997).

Ya hemos comentado que el hipotálamo y el sistema límbico están unidas a la neurofisiología de las emociones, sobre todo la «amígdala». Esto es algo común a los vertebrados. Pero su vínculo a partes más externas del córtex cerebral parece un rasgo evolutivo posterior, ligado a los primates y sobre todo a los humanos. La capacidad emocional estaría ligada a este rasgo 
común evolutivo con otras especies, pero el «contenido» y los factores que disparan la «emocionalidad» estarían progresivamente ligados al córtex como lugar del aprendizaje y la experiencia (LeDoux, 1998). Del mismo modo, hay claras evidencias de la implicación del hemisferio izquierdo también en la emoción, modulando el trabajo neurológico del hemisferio derecho, así como organizando las manifestaciones sociales de lo emocional (Carlson, 1999).

Las relaciones entre la emoción y lo cognitivo no son únicas, sino complejas. Desde el punto de vista neurológico, entran en juego complejísimas redes neuronales, poniendo en marcha multitud de áreas del cerebro, con funcionalidades complejas y variadas. Algunas teorías han insistido en que la emoción es resultado de cierto nivel de activación de la formación reticular del cerebro, una estructura compleja en el tallo del cerebro. En la medida en que la formación reticular sirve también para la integración virtual de toda actividad cerebral, la percepción o la acción estarían necesariamente imbuidos de contenido emocional. La mayoría de las veces las funciones cognitivas operadas en el neocórtex suelen ser activadas a partir de las límbicas, dando lugar a la evaluación y a la toma de conciencia emotiva. Por si fuera poco complejo, parece que estas mismas funciones cognitivas (recordar, pensar, juzgar, imaginar) pueden disparar ciertas emociones. Por todo ello se habla de un modelo de circuito doble que implica al sistema límbico y el neocórtex. En las primeras etapas de la ontogenia parece que el circuito subcortical es preferente y con el desarrollo progresivo de las capacidades cognitivas, se procesaría en ambos sentidos. Las relaciones ente ambas dimensiones mentales parecen ser, pues, enormemente estrechas. El sistema límbico también es fundamental para la memoria y el procesamiento de información (Laird et al., 1982).
Estos datos iluminarían el estéril debate sobre el afecto o emoción como variable independiente o no frente a lo cognitivo. Para los que dan preeminencia a lo afectivo, éste se contempla bien como un sistema amplificador de pulsiones innatas, ampliando la urgencia de algo, como un mecanismo de motivación central, desarrollado evolutivamente (Tomkins, 1981) o bien en el sentido de que «las preferencias no necesitan de las inferencias»: las emociones no necesitan de elaboracion cognitiva para suscitar respuestas viscerales y de actividad motora (Zajonc, 1984; cf. Goodale, 1982; Stephan et al., 1981). Sin embargo, es poco probable que la puesta en marcha de una reacción emotiva sea independiente de experiencias previas que impliquen cierto trabajo cognitivo (Lazarus, 1982) ${ }^{13}$. Desde luego, por lo menos para la experiencia afectiva, parece más que probable la existencia de algún proceso perceptivo/cognitivo, algún tipo de toma de conciencia (Izard, 1983; Harkness y Kilbride, 1983) e interpretación (Ginsburg y Harrington, 1996; Tomkins, 1981), siendo que el saber y la información también intervienen y modifican las emociones.

Izard (1984) propone un modelo de «bucle» («loop model») en donde cabrían dos sistemas, uno en el que la emoción sería un factor en el procesamiento de información y la cognición, y otro, en donde el sistema emotivo procesaría información «afectiva». Para Cicchetti y Schneider-Rosen (1985), las emociones pueden funcionar como contexto en el desarrollo cognitivo a la vez que la emergencia de nuevas emociones dependen del desarrollo cognitivo. Por su parte, Levy (1984) distingue entre dos niveles de conocimiento o saber («knowledge») en relación a la emoción. Uno denominado «de primer orden», vinculado a la memoria perceptual y esquemas sensorimotores y el otro, «de segundo orden», que referiría a una evaluación «cognitiva» que implicaría nombrar, clasificar, interpretar, dirigir, elementos todos ellos del sistema cultural del grupo. 
La incorporación de este elemento cognitivo permite a muchos autores distinguir entre emociones y sentimientos, incorporando estos últimos una toma de conciencia o experiencia subjetiva ${ }^{14}$. Para Rolls (1994) los sentimientos implican, desde el punto de vista neurocientífico, una toma de conciencia y alguna habilidad de procesamiento de tipo lingüístico. LeDoux (1995) distinguiría también entre emoción condicionada y la experiencia o sentimiento del mismo.

Desde las ciencias cognitivas, los teóricos de la modularidad de la mente (Fodor, 1983; Sperber, 1994) y del conexionismo (Churchland y Churchland, 1987) y de la especificidad de dominios cognitivos que citábamos en el apartado anterior nunca se han incluido a las emociones dentro de los módulos mentales innatos, precisamente por el mantenimiento que hacen del dualismo entre racionalidad y emocionalidad en el ser humano. Los modelos computacionales de la mente y la identificación del cerebro como procesador central, en sus distintas versiones más o menos conexionistas, han trabajado exclusivamente los productos proposicionales de la actividad mental como única fuente de conocimiento. Éstos son producto de las capacidades intelectuales de más alto nivel («higher order») de nuestro cerebro, que aparentemente no involucran actividad fisiológica alguna, al contrario que las emociones (Averill, 1996b).

Desde ese modelo integrado de la persona que citábamos antes, lo llamado a menudo «subjetivo - las motivaciones, los deseos e intenciones (Searle, 1990), las emociones - también son parte de su actividad psíquica y mental, inextricablemente unido a los procesos experienciales y del saber.

D’Andrade (1981) ve las emociones y sentimientos como respuestas internas sobre cómo van las cosas en relación a una variedad de clases de necesidades. Los sentimientos y las emociones nos dicen cómo es el mundo de una manera vívida («qualia», «mío»), haciendo incrementar la activación de varios esquemas para la acción y evaluación, permitiendo mientras tanto una demora de modo que la planificación, la secuencialidad de objetivos, el reconocimiento y otros procedimientos complejos pueden operar. Para él se trata de un sistema de información en «suspense» («information holding system») donde las emociones son bucles reverberantes que mantienen información de forma activa de modo que no desaparece, que permite una demora pero que trabaja contra el olvido. Las emociones y el razonamiento no son incompatibles, sino que se vinculan en un sistema de procesamiento total de información y de la construcción de sentido. Mediante la fusión del hecho que se describe y la reacción evaluativo-afectiva, los esquemas culturales consiguen tener un impacto direccional poderoso como valores implícitos (D’Andrade, 1981). Vandamme (1988) subraya que la emoción permite concentrar la atención y la energía en ciertos aspectos de la situación, propiciando, por medio de la simbolización, su organización, reorganización y jerarquización. La cognición sería una «metaemoción», un nivel organizativo superior por medio del cual se estructura y opera en lo simbólico, incrementando nuestra eficacia y resolución en el entorno, algo ya iniciado en el nivel de lo emocional. Esto no impediría a lo cognitivo cierto nivel de autonomía y feedback en lo emocional.

El afecto hace que algo sea importante, saliente ${ }^{15}$, involucrando al sujeto y orientándolo a la acción. El conocimiento, como saber, es algo más que el conocimiento proposicional desencarnado. El significado, precisamente, se constituye «valorativamente» y se engarza en el sujeto, involucrándolo, por medio de la intención, la motivación, el deseo, y las emociones que éstos vehiculan. Como Lave propone, la cognición en la acción está fusionada con 
el sentimiento desde el momento en que no puede separarse de la experiencia y la creación de valor (Lave, 1988). La muestra de los afectos no ha de entenderse como una expresión catártica de los sentimientos, sino más bien como declaraciones de la mente, la motivación y la intención (Schieffelin, 1983). Gell (1996) se refiere al «amor», con sus componentes de intimidad, disimulo y secreto, como un tipo particular de inteligencia que nos distingue, como una manera de adquirir conocimientos, de obtener, distribuir y transformar informaciones que son de un valor fundamental.

Por su parte, Damasio (1994) también ha subrayado, en su crítica a la imagen racionalista cartesiana del hombre y a la teoría de la elección racional reelaborado luego por el modelo de la «microeconomía», la importancia de la emoción y la evaluación afectiva en la toma de decisiones, subrayando saliencias, direccionalidades, propósitos. Además, las emociones también intervienen en la creatividad humana (Isen et al., 1987) y fijan la experiencia (entre ésta el dominio cognitivo) en la memoria. La crítica feminista y su recuperación del cuerpo ha puesto también el acento sobre la mutua constitución entre emociones y proceso de razonamiento (Jaggar 1989, citado en Williams, 2001) ${ }^{16}$.

Devereux (1979) establece una interesante conexión entre emoción, cognición e implicación («involvement») con la realidad a través de la simbolización y la fantasía, una capacidad que convierte a la «realidad» en algo muy multidimensional. La emocionalidad es básica en la construcción, también, de la función simbólica (Bates, 1979; Pinol-Douriez, 1987). Igual que la relación entre los objetos y sus nombres son experimentados por los niños en sus primeros años de vida en términos de contigüidad entre sí y con los contextos pragmáticos en que estas relaciones se producen, lo emocional es parte de las relaciones objetuales mediadas socialmente (Bates, 1979). Las emociones son vehículos por medio de los cuales los niños son integrados en las actividades y mundos comprensivos («understandings») de los adultos (Lutz, 1983).

La teoría del vínculo (Bowlby, 1969 , 1973; Ainsworth et al., 1974) especifica otra conexión con el conocimiento. Su operatividad como plataforma de seguridad emocional a partir de la cual el bebé desarrolla su curiosidad y capacidades explorativas protegiéndose de lo extraño indica algún tipo de conexión entre algunos procesos cognitivos y afecto. Las evidencias etológicas así parecen confirmarlo también ${ }^{17}$. Cicchetti y Schneider-Rosen (1985) describen algunas de las consecuencias cognitivas negativas en la relación afectiva anómala entre padres y niños severamente discapacitados y, por supuesto, en el caso de maltrato infantil.

El marco dialógico e interactivo de la socialización y aprendizaje humanos, a pesar de su variabilidad crosscultural en términos de contextos, intensidad, participación, direccionalidad y actores (cf. Ochs y Schieffeln, 1984; Harkness y Super, 1983; Grossmann et al., 1985; Sagi et al., 1985; Bretherton et al., 1979; Fraiberg, 1974) ha sido subrayado por infinidad de autores (Kaye, 1977; Charney, 1980; Schaffer, 1992; Butterworth, 1996; Brazelton y Tronick, 1980; Bretherton, 1985; Messer y Collis, 1996). El aprendizaje en el contexto social por medio del «andamiaje» («scaffolding») y presentación estructurada y «troceada» («chunks») (Schank y Abelson, 1977) de formatos y contenidos proporcionados por parte de los cuidadores, mediante la observación activa en un contexto de maestro-aprendiz (Bloch, 1991) o el descubrimiento «guiado» (D’Andrade, 1981), en la praxis (Lave, 1988), a partir de una «pedagogía» ausente en otros primates (Premack, 1984), en donde el infante es co-ayudado a organizar sus emociones y aprende los formatos de 
su expresión, es como se produce la socialización y el aprendizaje en las nuevas generaciones en la especie humana. Esta forma particular de interacción cooperativa y diálogo prelingüístico es capital en el establecimiento y mantenimiento de la intersubjetividad y la compartición y comprensión de las intenciones, sentidos y emociones de los demás, tan típicamente humanos.

Hoffman subraya la importancia de las emociones desde el punto de vista de la cognición social y el lugar de ésta en la cognición en general. El afecto juega un papel esencial en la distinción entre la comprensión de las personas y la comprensión de los objetos, aludiendo una diferencialidad de dominio entre la inteligencia física o mecánica y la social ${ }^{18}$. Para M. L. Hoffman (1981) la cognición social precede a la cognición no social, tanto es así que la permanencia de la persona es considerada anterior a la permanencia del objeto y esto gracias al involucramiento emocional respecto del primero, ausente en la relación con el segundo. El efecto positivo de la emoción en la memoria puede explicar este hecho. Pero también arguye que el principio de causalidad, vía reconocimiento de intencionalidad y estados mentales, así como de violaciones de las reglas sociales, es comprendido antes en la esfera de lo social. La conducta expresiva — gestos, posturas, elementos ilocucionarios del discurso- tiene consecuencias a la hora de activar y regular la experiencia de la emoción en otros, porque comunica intenciones, motivaciones, deseos. La empatía (Hoffman, 1981; Harris et al., 1989; Liebowitz, 1983), o emoción vicaria, el ponerse en el lugar del otro como ya insistía G. H. Mead (1967), asumir las emociones del otro gracias a la estructuración semejante entre el actor (sujeto) y el modelo (objeto), juega un gran papel en el reconocimiento del estado mental del otro y de sus condiciones, favoreciendo la cognición social y la correcta interpreta- ción de la situación, con los consiguientes efectos «estructurantes» sobre la relación social (cf. infra).

Lo interesante de esta perspectiva, apoyada por una abundante literatura al respecto, tanto en psicología del desarrollo como en primatología, es la relación entre el reconocimiento y expresión significativa de las emociones y una teoría mental sobre los otros. Las teorías de la referencia social («social referencing») (Campos y Stenberg, 1981; Klinnert et al., 1983) mencionan cómo el/la niño/a busca información sobre los estados mentales de la madre (o cuidador/a) por medio de la interpretación de su cara y la expresión de sus emociones, regulando su propia conducta, conecta emoción, cognición y comportamiento. $\mathrm{La}$ capacidad de «provocar» emociones (consolar, molestar, fastidiar), están al alcance del niño a partir del segundo año de vida, progresando su interés sobre el estado emocional de los demás a partir del tercer año (Dunn, 1988). Algunos, desde una orientación maquiavélica de la inteligencia social (Whiten y Byrne, 1988) llegan a opinar que las emociones, más que como signos de una intención de actuar, deberían comprenderse como intentos de manipular el comportamiento de otros mediante la información de que ciertos tipos de comportamiento van a ocurrir (Fridlund y Duchaine, 1996). Por su parte, Bates (1979) insiste en que la resolución de tareas, la enseñanza y la empatía -que incluye la detección y participación en estados emocionales- están ligados desde el punto de vista ontogenético en el niño normal. Baron-Cohen (1991) subraya, en ese mismo sentido, la ligazón entre las incapacidades en competencia social -entre ellas una teoría de la mente-, en destrezas comunicativas y en «prentensión» («pretence») en los niños autistas.

A este respecto, lejos de una inteligencia piagetiana general, podríamos hablar de una suerte de «confluencia» («conflation». Cf. Johnson, 1997, cit. en Lakoff y 
Johnson, 1999) entre emoción y cognición, como experiencia «holística» crosmodal en la infancia, cuyos planos de relación y mutua constitución variarían —dando lugar a subsiguientes procesos de especialización de dominios - en relación a futuros estadios de maduración neurológica y ontogenética (Gibson, 1996), a la estructuración propia de las tareas cognitivas (mentales) en términos de su construcción progresiva hasta que se convierten en automáticas y rutinarias (Bates, 1979: et al., 1988) y a las variadas tecnologías educativas y de socialización.

No podemos extendernos aquí a discutir las teorías de la crossmodalidad y de la metáfora. Sólo apuntaremos la relevancia de la inteligencia social $-\mathrm{y}$ parte de ella una teoría sobre la mente y dentro de ésta, de la emocionalidad-y su operacionalización en otros dominios cognitivos, en la evolución de las habilidades cognitivas de nuestra especie durante el proceso de sapientización (cf. Jolly, 1972; Goody, 1995; Whiten, 1991; Byrne, 1995). En ello, la atribución de deseos, intenciones y emociones marca, según Jolly (1996), el largo camino hacia la simbolización y la cultura: «A lo largo de milenios de evolución, las mentes progresaron desde "ese mono quiere morderme", a "no le gusto a ese primate", a "ese trueno quiere asustarme", a "Zeus está enfadado conmigo"» (traducción propia) (Nota. Sobre teorías «naturalistas» de la religión, cf. Boyer, 1994).

\section{Efectos emocionales de lo social, efectos sociales de la emoción}

Desde una aproximación macrológica, es claro que la estructura social determina y gestiona perfiles y conductas emocionales. La socialización diferencial en el afecto tiene que ver con esto (Miller y Sperry, 1987). Un ejemplo clásico es cómo las familias de clase media preparan a sus hijos para controlar sus emociones más que las familias de clase trabajadora, de forma que cada clase prepara a sus hijos en un perfil psicológico atribuido como propio (Hochschild, 1979). Diferencias en los contenidos emocionales y formas expresivas de las co-narraciones entre padres e hijos pueden justificarse en parte por diferencias de clase social (Burger y Miller, 1999) y también por la estructura de la unidad doméstica (Seymour, 1983). Las variaciones históricas e ideológicas también producen variaciones en los estilos emocionales, como Demos (1996) muestra para una Nueva Inglaterra prerrevolucionaria, donde la vergüenza es un sentimiento predominante a la Nueva Inglaterra colonial dominada por la culpa. La posmodernidad y sus fuerzas producen una determinada gestión de las emociones (Hochschild, 1983).

No podemos desligar las emociones de sus vínculos morales, de la propiedad o no de sus expresiones socialmente construidas y legitimadas, de sus lazos con las instituciones, normas y valores que las informan (Harkness y Super, 1983). En esa medida, las emociones tienen siempre un referente constitutivo público, compartido, una comunidad para quien y por quien las emociones adquieren su sentido. Las emociones no pueden tomarse abstractamente de las experiencias que las constituyen, por mucho que se «localicen» en el self como lugar de las mismas (Harris, 1989), sino que implican también una visión sobre el mundo y lo social, unido a una praxis socialmente guiada por medio de reglas, instituciones, valores. Emociones «complejas» como la vergüenza y la culpa remiten, en última instancia, a regulaciones del self en y por lo social (Fajans, 1983; Solomon, 1984), a la comprensión de la agencia y la responsabilidad, a códigos de conducta y etiqueta, participación y aprobación social, sanciones y definiciones sobre la falta, la transgresión y la restitución social, un orden moral y ético, al fin y a cabo (Rosaldo, 1983; Schieffelin, 1983; Lutz, 1983; Scheff, 1990). A la inversa, no sólo 
las emociones aparecen en relación a juicios evaluativos sobre el orden de las cosas, sino que se puede hablar, también, de una propiedad y corrección de las emociones (Williams, 2001), de un orden moral para las emociones.

Lo emocional tiene, a su vez, «efectos» sociales, consecuencias «estructurantes» para lo social (Williams, 2001; Gordon, 1990). Puede hablarse de la «eficacia» social de las emociones (Barbalet, 1998) en el mismo sentido en que C. Lévi-Strauss (1977) decía sobre lo simbólico, tanto desde el punto de vista de la re-creación del orden social como del de su cambio. E. Durkheim (1982) atribuía a la emocional una causalidad directa en la solidaridad mecánica, en la vinculación directa e inmediata del individuo con la colectividad. La «efervescencia» colectiva tiene que ver con esto, tal como también subrayó V. Turner en su diferenciación entre estructura y comunitas en los procesos rituales (Turner, 1977), vinculada esta última a los procesos de renovación social, como revoluciones y revitalizaciones religiosas. La comunicación emocional es crucial en el mantenimiento de las lealtades y vínculos grupales (D'Andrade, 1981; Wentworth y Yardley, 1994). M. Rosaldo (1983), en su trabajo sobre los Illongots de Filipinas y sus específicas metáforas para lo emocional, destaca cómo la vergüenza ordena las relaciones sociales, evitando que la ira destruya los lazos cooperativos entre los parientes. Para los Ifaluk, de las Islas Carolina en Micronesia, las emociones están inscritas en el corazón de sus interacciones interpersonales sociales y, lejos de referirse a estados mentales internos, les proporciona el marco motivacional para el mantenimiento de su sistema de rango y la obediencia y cooperación que acompañan a éste (Lutz, 1983).

Desde el punto de vista de la interacción social, las emociones se trabajan y retrabajan jerárquica y linealmente (Ginsburg y Harrington, 1996) permitiendo un con- tinuo de la relación en el tiempo. Levy (1984), en su estudio sobre las emociones en Tahiti, señala que expresar una emoción es una declaración a los otros sobre la relación del actor con su ejecución social en acción («ongoing social performance»). Es un «metamensaje» sobre la relación del actor con su comportamiento socialmente codificado. Este aspecto eminentemente expresivo y comportamental, pragmático, permite a algunos, de nuevo, distinguir entre emociones y sentimientos (Schieffelin, 1983; Fajans, 1983).

Por otro lado, las emociones y sentimientos dan forma colectiva a la experiencia e identidad de grupo también por medio de la transmisión de narraciones, expresiones y metáforas (James, 1997), pero también de chismes y rumores como en los Kaluli de Papua (Nueva Guinea) (Schieffelin, 1983). El uso de las emociones, unido a determinados trabajos sobre el cuerpo, es intrínseco a la mayoría de los rituales de paso, residiendo en ello buena parte de la «eficacia» social de los mismos (Turner, 1980; Whitehouse, 1996). Hart cuenta cómo en los Tiwi (norte de Australia), la teología, los arcanos, el mundo sagrado y natural (cosmología, geografía, biología, etc.), la «herencia cultural completa de la tribu», es comunicado, enseñado, en contextos de fuerte experiencia afectiva y emotiva, con un trabajo determinado sobre el cuerpo (posturas, prescripciones alimenticias, contactos regulados, horarios y dietas fijas) (Hart, 1963).

\section{Emoción y experiencia: teorías del cuerpo}

Ya para terminar, en nuestro intento de reubicar el debate en relación a paradigmas más complejos y articulados sobre lo humano y lo social, vamos a referirnos a las teorías del cuerpo («embodiment») y su concepción integrada del sujeto y su experiencia. Es una 
perspectiva ligada principalmente a la fenomenología de Merleau-Ponty ${ }^{19}$, aunque no exclusivamente (Putnam, 1981, 1999) ${ }^{20}$. Desde el punto de vista fenomenológico, las emociones se entienden como una forma de estar y ser en el mundo. Y es el cuerpo el instrumento original con el que los humanos dan forma a su mundo a la vez que la sustancia original a partir de la cual el mundo humano es formado (Mauss, 1950). Éste es precisamente uno de los principios básicos de una concepción encarnada de la experiencia humana: uno vive desde el cuerpo y lo sensible, no puede mantenerse más la oposición mente/cuerpo.

Pero este cuerpo no es «dado», en el sentido del individualismo o del organicismo. Es un cuerpo que se construye histórica, cultural y biográficamente a partir de aquellas posibilidades y aperturas neurofisiológicas y cenestésicas de las que hablábamos anteriormente. Reubicar la experiencia - siempre social en cuanto que construida socialmente- en el cuerpo devuelve la mente, lo psicológico, y lo cultural a sus dimensiones biosociales, algo olvidadas por los relativistas y constructivistas. Lo físico, psíquico y social forman partes articuladas del complejo proceso de la experiencia.

Sin hacer un repaso exhaustivo del tema en Antropología, recordaremos simplemente que Lévi-Bruhl fue uno de los primeros antropólogos en destacar la íntima relación entre lo conceptual, lo sensible, lo emocional y la actividad corporal. A partir de conceptos como «la ley de participación», característica de una mentalidad pre-lógica (no en el sentido evolutivo) en donde existen correspondencias y relaciones donde la lógica encontraría oposiciones y contradicciones, insiste en la vivencia colectiva e individual de los conceptos no de forma abstracta, sino experiencial, concreta (Shore, 1996). La «mentalidad» (mentality) reside en la intersección de la sensorialidad humana común y un conjunto variable de representaciones culturales o modelos [Nota. No es que el pensamiento «prelógico» sea característico exclusivo de las sociedades y mentalidades «primitivas», sino que las representaciones colectivas de algunos grupos legitiman el pensamiento místico («participatorio» y emocional) en una cantidad de contextos más amplia que otros grupos. Sólo con la aparición de lo que denominó «conciencia individual» estos conceptos que asumen la contradicción en sí mismos derivan en conceptos lógicos y abstractos, dando lugar a relaciones abstractas de identificación simbólica, similaridad o metáfora (Shore, 1996). V. Turner (1980) es otro que ha destacado la importancia de lo sensorial y corpóreo en los procesos de simbolización y ritualización (Turner, 1980).]

Aunque exista una gran variedad crosscultural sobre los distintos usos del cuerpo en relación a la experiencia y expresión de las emociones (Peixoto, 1995; Bozon y Heilborn, 1996), aunque podamos matizar la necesaria y única correspondencia neurofisiológica a determinados estados emocionales, lo cierto es que toda experiencia está inscrita y a la vez mediada por el cuerpo.

A partir de estas premisas, y para terminar después de tantas idas y venidas, permítasenos la petulancia de ensayar una definición, por si el lector tiene la sensación de que los árboles no le han dejado ver el bosque. Resumiendo hasta aquí, podríamos entender la emoción (los procesos emocionales) como un campo constitutivo/constituido de la experiencia encarnada («embodied») de un sujeto biopsicológico-social construido sociohistórica y políticamente a partir de diversas ideologías - morales - y tecnologías educativas y del cuerpo a lo largo de la continua ontogenia del ciclo vital humano. Las emociones permiten al sujeto dar valor, saliencia y significación (subjetiva e intersubjetiva) a su relación constitutiva con el mundo, implicándolo, engarzándolo a él. Se trata de un sujeto que siente con otros sujetos que también sienten (Denzin, 1984), compartiendo 
y negociando significados sobre lo que activamente le sucede. Las dimensiones «pragmáticas» y comunicativas (Schieffelin, 1983) de lo emocional (Papataxiarchis, 1994; Williams, 2001; Crossley, 1998, en Williams, 2001), son arte y parte del proceso por medio del cual las personas, los sujetos sociales, los grupos, construyen y son construidos por su entorno, siempre social. Ésta es la «irracionalidad» que nos cualifica frente a otros parientes próximos y lejanos; es ésa, entre otras, la condición y el producto de nuestra humanidad.

\section{REFERENCIAS}

Abu-Lughod, L., y Lutz, C. (1990), «Introduction: emotion, Discourse and the Politics of Everyday Life», en ABu-LuGHOD, L. y LuTz, C. (eds.), Language and The Politics of Emotion, Cambridge, CUP.

Ainsworth, M. D.; Bell, S. M., y Stayton, D. J. (1974), «Infant-mother attachment and social development: socialisation as a product of reciprocal responsiveness to signals», en Richards, M. P. (ed.), The integration of a child into a social world, Cambridge, CUP, pp. 99-135.

Atran, S. (1990), Cognitive Foundations of Natural History. Towards an anthropology of science, Cambridge, Cambridge University Press.

- (1998), «Folk Biology and the Anthropology of Science: Cognitive Universals and Cultural Particulars», Behavioral and Brain Sciences, núm. 21, pp. 547-609.

AveriLl, J. R. (1996a), «Intellectual Emotions», en Harré, R., y Gerrod Parrott, W. (eds.), The Emotions.Social, Cultural and Biological Dimensions, Londres, SAGE, pp. 24-38.

- (1996b), «An Analysis of Psychophysiological Symbolism and its Influence on Theories of Emotion», en HARRÉ, R., y Gerrod Parrott, W. (eds.), The Emo- tions. Social, Cultural and Biological Dimensions, Londres, SAGE, pp. 204-228.

Barbalet, J. M. (1998), Emotion, Social Theory and Social Structure. A Macrosociological Approach, Cambridge, CUP.

Barkow, J.; Cosmides, L., y Tooby, J. (eds.) (1992), The adapted mind: evolutionary psychology and the generation of culture, Nueva York, Oxford University Press.

BARon-CoHEN, S. (1991), «Do people with autism understand what causes emotion?», Child Development, núm. 62, pp. 385-395.

BAtEs, E. (1979), «Intentions, Conventions and Symbols», en Bates, E.; Benigni, L.; Bretherton, I.; Camaioni, L., y VolTERRA, V., The Emergence of symbols. Cognition and Communication in Infancy, Nueva York, Academic Press, pp. 33-68.

Bates, E.; Bretherton, B., y Snyder, L. (1988), From first words to grammar. Individual differences and dissociable mechanisms, Cambridge, CUP.

Bateson, G. (1973), Steps on an ecology of mind, St Albans, Paladin.

Berlin, B., y Kay, P. (1991) (1969), Basic Color Terms: Their Universality and Evolution, Berkeley, University of Color Terms.

Bloch, M. (1991), «Language, Anthropology and Cognitive Science», en Man NS, 26, pp. 183-198.

BOyer, P. (1994), The Naturalness of Religious Ideas: A Cognitive Theory of Religion, Berkeley, University of California Press.

Bowlby, R. (1969), Attachment and Loss, vol. 1, Attachment, Londres: Hogarth Press.

- (1973), Attachment and Loss, vol. 2, Separation, Londres, Hogarth Press.

Bozon, M., y Heilborn, M. L. (1996), «Les caresses et les mots. Initiations amoureuses à Rio de Janeiro et à Paris», Terrain, núm. 27, pp. 37-58. 
Brazelton, T. B., y Tronick, E. (1980), «Preverbal communication between Mothers and Infants», en Olson, D. R. (ed.), The Social Foundations of Language and Thought. Essays in Honor of Jerome S. Bruner, Nueva York, Norton, pp. 299-315.

Bretherton, I. (1985), «Attachment theory: Retrospect and Prospect», en Bretherton, I., y Waters, E., Growing points in attachment theory, Monographs for the Society for Research in Child Development, serial núm. 209, vol. 50 (1\&2), pp. 3-35.*

Bretherton, I., Bates, E.; Benigni, L.; Camaioni, L., y Volterra, V. (1979), «Relationships between Cognition, Communication and Quality of Attachment», en BATES, E., et. al., The Emergence of symbols. Cognition and Communication in Infancy, Nueva York, Academic Press, pp. 223-269.

Bruner, J. (1974), «The organization of early skilled action», en RichARDS, M. P. M. (ed.), The integration of a child into a social world, Cambridge, CUP, pp. 167-184.

Bruner, J.; Greenfield, P., y Olver, R. (1966), Studies in cognitive growth, Nueva York, Wiley.

BRUNER, J. (1996), «Frames for thinking. Ways of Making Meaning», en Olson, D. R., y Torrance, T. (eds.), Modes of Thought. Explorations in Culture and Cognition, Cambridge, CUP, pp. 93-105.

Burger, L. K., y Miller, P. G. (1999), «Early talk about the past revisited: affect inworking-classand middle-calss children»s co-narrations», Journal of Child Language, núm. 26, pp. 133-162.

Butterworth, G. (1996), «The origins of language and thought in early childhood», en Lock, A., y Peters, Ch. P. (eds.), Handbook of Symbolic Evolution, Oxford, Oxford Science Publication, pp. 469-482.

Byrne, R. (1995), The thinking ape: evolutionary origins of intelligence, Oxford, Oxford University Press.
Byrne, R., y Whiten, A. (eds.) (1988), Machiavellian intelligence: social expertise and the evolution ofintellect in monkeys, apes, and humans, Oxford, Clarendon Press.

Cacioppo, J. T., y Petty, R. E. (1981), «Lateral asymmetry in the expression of $\operatorname{cog}$ nition and emotion», Journal of Experimental Psychology: Human Perception and Performance, núm. 7, pp. 333-341.

Campos, J. J., y Stenberg, C. R. (1981), «Perception, appraisal and emotion: The onset of socialreferencing», en LAmb, M. E., y Sherrod, L. R. (eds.), Infant social cognition, Hillsdale, N. J.: Erlbaum, pp. 273-314.

Calvin, W. H. (1997), How brains think, Londres, Phoenix.

Carlson, N. R. (1999), Fisiología de la Conducta, Barcelona, Ariel.

Changeux, J. P. (1986), Neuronal Man, Nueva York, Oxford University Press. Churchland, P. M., y Churchland, P. M. (1990), «Could a Machine Think?», Scientific American, núm. 262 (1) pp. 26-31.

Cicchetti, D., y Schneider-Rosen, K. (1985), «Relationship between affect and cognition in atypical infansts», en IZARD, C. E.; Kagan, J., y ZaJonc, R. B. (eds.), Emotions, cognition, and behavior, Nueva York, Cambridge University Press, pp. 366-406.

Crapanzano, V. (1994), «Réflexions sur une anthropologie des émotions», Terrain, núm. 22, marzo, pp. 109-117.

Crossley, N. (1998), «Emotions and communicative action», en Bendelow, G., y Williams, S. J. (eds.), Emotions in social life: Critical Themes and Contemporary Issues, Londres, Routledge.

Csordas, Th. (1994), «Introduction: the body as representation and being-in-the-world», en Csordas, Th. (ed.), Embodiment and Experience. The existential ground of culture and self, Cambridge, CUP, pp. 1-24. 
Damasio, A. R. (1994), Descartes» Error: Emotion, Reason and the Human Brain, Nueva York, Putnam.

D'ANDRADE, R. (1981), «The cultural part of cognition», Cognitive Science, núm. 5, pp. 179-195.

- (1992), «Shemas and motivation», en D'Andradre, R., y Strauss, C., Human Motives and cultural models, Cambridge, CUP, pp. 23-44.

DARwin, Ch. (1965) (1872), The Expression of the Emotions in Man and animals.

DAVIDSON, R. J. (1984), «Affect, cognition, and hemispheric specialization», en IZARD, C. E.; Kagan, J., y Zajonc, R. B. (eds.), Emotions, cognition, and behavior, Nueva York, Cambridge University Press, pp. 320-365.

Demos, J. (1996), «Shame and Guilt in Early New England», en Harré, R., y Gerrod Parrott, W. (eds.), The Emotions. Social, Cultural and Biological Dimensions, Londres, SAGE.

Denzin, N. K. (1984), On Understanding Emotion, San Francisco, Jossey Bass.

Devereux, G. (1979), «Fantasy and symbol as dimensions of reality», en НоOK, R. H. (ed.), Fantasy and symbol, Nueva York, Academic Press, pp. 19-32.*

Donald, M. (1991), Origins of the Modern Mind: Three States in the Evolution of Culture and Cognition, Cambridge, Mass., Harvard University Press.

Dreyfus, H. (1979) (1972), What computers can't do, Nueva York, Harper and Row.

Dunn, J. (1988), The Beginnings of Social Understanding, Oxford, Basil Blackwell.

Dunn, J.; Brown, J., y Beardsall, L. (1991), «Family talk about emotions, and children's later undertanding of other's emotions», Developmental Psychology, núm. 27, pp. 448-455.

DurkHEIM, E. (1982) (1912), Las formas elementales de la vida religiosa, Madrid.

Durkheim, E., y Mauss, M. (1963), Primitive Classification, en NEEDHAM, R. (ed.), Londres, Cohen \& West.
Edelman, G. M. (1992), Bright air, brilliant fire, Londres, Allen Lane.

Eibl-Eibesfeldt, I. (1993), Biología del comportamiento humano. Manual de etología humana, Madrid, Alianza.

Ekman, P. (1979), «About brows: emotional and conversational signals», en VON Cranack, M.; Foppa, K.; Lapenies, W., y Ploog, D. (eds.), Human ethology: claims and limits of a new dicipline, Cambridge, CUP, pp. 169-202.

- (1980), The Face of Man. Expressions of Universal Emotions in a New Guinea Village, Nueva York, Garland STPM Press.

- (1992), «An argument for basic emotions», Cognition and Emotion, VI, pp. 169-200.

Elias, N. (1982) (1939), The Civilizing Process, Oxford, Basil Blackwell.

ERDI, P. (1988), «From Brain theory to future generations computer systems", en Carvallo, M. E. (ed.), Nature, Cognition and System I, Dordrecht, Kluwer Academic Publishers, pp. 77-94.

FAJANS, J. (1983), «Shame, Social Action, and the Person among the Baining», en Ethos, 11:3, 166-180.

FAVRET-SAADA, J. (1994), «Weber, les émotions et la religion», Terrain, núm. 22, marzo, pp. 93-108.

Fodor, J. (1983), The modularity of mind, Cambridge Mass., MIT Press.

Fraiberg, S. (1974), «Blind Infants and Their Mothers: An Examination of the Sign System», en Lewis, M., y RosenBLum, L. A. (eds.), The Effect of the Infant on its Caregiver, Nueva York, JohnWiley \& Sons, pp. 215-232.

Freeman, W. J. (1991), «The physiology of perception», Scientific American, febrero, pp. 34-41.

Freeman, W. J., y Skarda, C. (1987), «How Brains make chaos in order to make sense of the world», Brain and Behavioral Science, 10, 2, pp. 161-195.

Freund, P. E. S. (1988), «Understanding socialized human nature», Theory and Society, núm. 17, pp. 839-864. 
Fridlund, A. J., y Duchaine, B. (1996), «Facial Expressions of Emotion» and the Delusion of the Hermetic Self», en Harré, R., y Gerrod Parrott, W. (eds.), The Emotions. Social, Cultural and Biological Dimensions, Londres, SAGE.

Foucault, M. (1988), «Technologies of the self», en Martin, L. H.; Gutman, H., y Hutton, P. H. (eds.), Technologies of the self: A Seminar with Michel Foucault, Londres, Tavistock.

Gardner, H. (1983), Frames of mind. The theory of multiple intelligences, Nueva York, Basic Books.

Geertz, C. (1973), The Interpretation of Cultures, Nueva York, Basic Books.

Gell, A. (1996), «Amour, connaissance et disimulation», Terrain, núm. 27, pp. 5-14.

Gibson, K. (1996), «The ontogeny and evolution of the brain, cognition, and language», en Lock, A., y Peters, Ch. R. (eds.), Handbook of Symbolic Evolution, Oxford, Oxford Science Publication, pp. 407-431.

Ginsburg, G. P., y Harrington, M. E. (1996), «Bodily States and Context in Situated Lines ofAction», en HARRÉ, R., y Gerrod Parrott, W. (eds.), The Emotions. Social, Cultural and Biological Dimensions, Londres, SAGE, pp. 229-258.

Goldschmidt, W. (1993), «On the relationship between Biology and Anthropology», Man (NS), núm. 28, pp. 341-359.

GómEz, J. C., y NúÑEz, M. (1998), «La Mente social y la mente física: desarrollo y dominios de conocimiento», Infancia y Aprendizaje, núm. 84, pp.

Goodale, M. A. (1982), "Vision as a sensorimotor system», en RoBinson, T. E. (ed.), $A$ behavioralapproach to brain research, Nueva York, Oxford University Press, pp. 41-61.

Gordon, S. (1990), «Social structural effects on emotions», en KEMPER, T. (ed.), Research Agendas inthe Sociology of Emotions, Nueva York, State University of New York Press.

Goody, E. (1995), «Introduction: Some implications of a social origin of intelligence», en Goody, E. N. (ed.), Social Intelligence and Interaction, Cambridge, CUP, pp. 1-33.

Greenberg, L. S., y Safran, J. (1987), «Emotin, Cognition and Action», en Eysenck, H. J., y Martin, I. (eds.), Theroetical Foundations of Behavior Therapy, Nueva York, Plenum Press, pp. 295-311.

Gumperz, J. J., y Levinson, S. C. (1991), «Rethinking linguistic relativity», Current Anthropology, núm. 32, pp. 613-623.

HARAway, D. J. (1990), «Investment Strategies for the Evolving Portfolio of Primate Females», en JAcobus, M.; Fox Keller, E., y Shuttleworth, S. (eds.), Body/Politics: Women andthe Discourse of Science, Nueva York, Routledge, pp. 139-162.

- (1991), Simians, Cyborgs and Women: The Reinvention of Nature, Nueva York, Routledge.

Harkness, S., y Super, Ch. M. (1983), «The Cultural Construction of Child Development: A Framework for the Socialization of Affect», Ethos, 11, 4, pp. 221-231.

Harkness, S.; Super, Ch. M., y Kilbride, Ph. L. (1983), «Introduction: The socialization of Affect», Ethos, 11:4, pp. 215-220.

Harlow, H. F., y Harlow, M. K. (1962), «Social Deprivation in Monkeys», Scientific American, núm. 207, pp. 136-146.

Harris, G. G. (1989), «Concepts of Individual, Self, and Person in Description and Analysis», American Anthropologist, núm. 91, pp. 599-612.

Harris, P. L.; Johnson, C. M.; Hutton, D.; Andrews, G., y CoOK, T. (1989), "Youngchildrens theory of mind and emotion», Cognition and Emotion, núm. 3. 
Hart, C. W. M. (1963), «Contrasts between Prepubertal and Postpubertal Education», en SPIndler, G. (ed.), Education and Culture, Nueva York, Holt, Rinehart \& Winston.

Heelas, P. L. F. (1996), «Emotion Talk across Cultures», en Harré, R., y Gerrod Parrott, W. (eds.), TheEmotions. Social, Cultural and Biological Dimensions, Londres, SAGE, pp. 171-199.

Herrman, D. J., y Raybeck (1981), «Similarities adn Differences in Meaning in Six Cultures», Journal of Cross-Cultural Psychology, núm. 12, pp. 194-206.

Hochschild, A. (1979), «Emotion work, feeling rules, and social structure», American Journal of Sociology, núm. 85, pp. 551-575.

- (1983), The Managed Heart: The Commercialization of Human Feeling, Berkeley, CA, University of California Press.

Hoffman, M. L. (1981) «Perspectives on the difference between underStanding people andunderstanding things: the role of affect», en Flavell, J. H., y Ross, L., Social Cognitive Development, Cambridge, CUP, pp. 67-81.

Hutchins, E., y Hazlehurst, B. (1995), «How to invent a shared lexicon: the emergence ofshared form-meaning mappings in interaction», en Goody, E. (ed.), Social Intelligence and Interaction. Expressions and Implications of the social bias in human intelligence, pp. 53-67.

Ingold, T. (1991), «Becoming Persons: Conciousness and Sociality in Human Evolution», Cultural Dynamics, núm. 4, pp. 355-378.

Isen, A. M.; Daubman, K. A., y Nowicki, G. P. (1987), «Possitive Affect Facilitates Creative Problem Solving», Journal of Personality and Social Psychology, 52, 6, pp. 1122-1131.

IzARD, C. E. (1979), «Emotions as motivations: an evolutionary-developmental perspective», en Dienstbier, R. A.,
Nebraska symposium on motivation 1978 , Lincoln, University of Nebraska Press. - (1983), «Emotions in Personality and Culture», Ethos, 11:4, pp. 305-312.

- (1984), «Emotion-cognition relationships and human development», en IZARD, C. E.; KAGAN, J., y ZAJONC, R. B. (eds.), Emotions, cognition, and behavior, Nueva York, Cambridge University Press, pp. 17-37.

IzARD, C. E., y Buechler, S. (1980), «Aspects of Conciousness and Personality in Terms of Differential Emotions Theory», en Plutchik, R., y Kellerman, H. (eds.), Emotion: Theory, Research and Experience, vol. I, Theories of Emotion, Nueva York, Academic Press., pp. 165-187.

JAGGAR, A. (1989), «Love and knowledge: emotion in feminist epistemology», en Bordo, S., y Jaggar, A. (eds.), Gender/Body/Knowledge: Feminist Reconstructions of Being and Knowing, Londres, Rutgers University Press.

JAMES, W. (1997), «The names of fear: memory, history and ethnography of feeling among Urdukrefugees», Journal of the Roy. Anthrop. Inst., 3,1, pp. 115-131.

Johnson, C. (1997), «Metaphor vs. Conflation in the Acquisition of Polysemy: The Case of SEE», en Hiraga, M. K.; Sinha, C., y Wilcox, S. (eds.), Cultural, Typological and Psychological Issues in Cognitive Linguistic, Current Issues in Linguistic Theory 152, Amsterdam, John Benjamins.

Johnson, M. (1987), The Body in the Mind: The Bodily Basis of Imagination, Reason and Recovering the Self, Londres, Meaning. Chicago, Chicago University Press. Jolly, A. (1972), The Evolution of Primate Behavior, Nueva York, Nueva York.

- (1996), «Primate communication, lies, and ideas», en Lock, A., y Peters, Ch. R. (eds.), Handbookof Human Symbolic Evolution, Oxford, Clarendon Press, pp. 166-177. 
KAGAN, J. (1984), «The idea of emotion in human development», en IZARD, C. E.; Kagan, J., y Zajonc, R. B. (eds.), Emotions, cognition, and behavior, Nueva York, Cambridge University Press, pp. 38-71.

KAYE, K. (1977), «Toward the origin of dialogue», en Schaffer, H. R. (ed.), Studies in mother-infant interaction, Londres, Academic Press, pp. 89-118*.

Kaye, K., Charney, R. (1980), «How mothers maintain "Dialogue" with Two-yer-olds», en Olson, D. R. (ed.), The Social Foundations of Language and Thought. Essays in Honor of Jerome S. Bruner, Nueva York, Norton, pp. 211-230.

Klinnert, M. D.; Campos, J. J.; Sorce, J. F.; EMde, R. N., y SveJdA, M. (1983), «Emotions asbehavior regulators: Social referencing in infancy», en PLUTCHIK, R., y Kellerman, H. (eds.), Theemotions, vol. 2, Emotions in early development, Nueva York, Academic Press, pp. 57-86.

Laird, J. D.; Wegener, J. J.; Halal, M., y Szegda, M. (1982), «Remembering what you feel: Theeffects of emotion on memory», Journal of Personality and Social Psychology, núm. 42, pp. 646-657.

LAKoff, G., y Johnson, M. (1999), Philosophy in the flesh. The embodied mind and its challenge to western thought, Nueva York, Basic Books.

LAtour, B. (1992) We have never been Modern, Nueva York, Harvester/Wheatsheaf.

Lave, J. (1988), Cognition in practice, Cambridge, CUP.

LAZARUs, R. S. (1982), «Thoughts on the relations between emotion and cognition», American Psychologist, núm. 37, pp. 1019-1024.

Leder, D. (1990), The Absent Body, Chicago, University of Chicago Press.

Ledoux, J. (1995), «Emotion: Cllues from the brain», Annual Review of Psychology, núm. 46, pp. 209-235.
- (1998), The Emotional Brain, Londres, Weidenfeld and Nicolson.

Levi-Strauss, C. (1977) (1958), Antropología estructural, Buenos Aires, Eudeba, «La eficacia simbólica», pp. 168-182.

Levy, R. I. (1984), «Emotion, knowing, and culture», en SHweder, R., y Levine, R. (eds.), Mind, Self, and Emotion, Cambridge, CUP, pp. 214-237.

Liebowitz, M. R. (1983), The Chemistry of Love, Boston, Little Brown \& Co.

Lock, A., y Colombo, M. (1996), «Cognitive abilities in a comparative perspective», en Lock, A., y Peters, Ch. R. (eds.), Handbook of Symbolic Evolution, Oxford, Oxford Science Publication, pp. 596-643.

Lupton, D. (1998), The Emotional Self, Londres, SAGE.

Lutz, C. (1982), «The domain of emotion words in Ifaluk», American Ethnologist, núm. 9, pp. 113-128.

- (1983), «Parental Goals, Ethnopsychology and the Development of Emotional Meaning», Ethos, 11:4, pp. 246-262.

- (1988), Unnatural Emotions, Chicago, Chicago University Press.

Lutz, C., y White, G. M. (1986), «The Anthropology of Emotions», Annual Review of Anthropology, núm. 15, pp. 405-436.

Maturana, H. R., y Varela, F. J. (1980), Autopoiesis and cognition: the recognition of the living, Dordrecht, Reidel.

- (1992), The tree of knowledge: the biological roots of human understanding, Boston, Londres, Shambhala.

Mauss, M. (1950), «Les Techniques du Corps», Sociologie et Anthropologie, París, PUF.

Mead, G. H. (1967), Mind, Self and Society, Chicago, Chicago University Press.

Messer, D., y Collis, G. (1996), «Early interaction and cognitive skills: implications for the acquisition of culture», en Lock, A., y Peters, Ch. R. (eds.), Handbook of Human Symbolic Evolution, Oxford, Clarendon Press, pp. 432-468. 
Miller, P. J., y Moore, B. B. (1987), «The socialization fo anger and agresion», Merril-Palmer Quarterly, 33 (I), pp. 1-31.

Mithen, S. (1996), The Prehistory of the Mind: the cognitive origins of art, religion and science, Londres, New York, Thames and Hudson.

Montagu, A. (1959), «Natural Selection and the Origin and Evolution of Weeping in Man», Science, núm. 130, diciembre, pp. 1572-1573.

Moore, C. C.; Romney, A. K., y Hsia, T.-L. (1999), «The Universality of the Semantic Structure of emotion Terms: Methods for the Study of Interand Intra-Cultural Variability», American Anthropologist, 10 (3), núm. 529-546.

Morin, E. (1973), Le paradigme perdu: la nature humaine, París, Le Seuil.

Ochs, E., y SchiefFelin, B. B. (1984), «Language acquisition and socialization. Three Developmental Stories and their implications», en SHWEDER, R., y LEVINE, R. (eds.), Culture Theory. Essays on Mind, Self, and Emotion, CUP, Cambridge, pp. 276-320.

Olson, D. (ed.) (1980), The Social Foundations of Language and Thought. Essays in Honor of Jerome S. Bruner, Nueva York, Norton, pp. 90-108.

Osgood, Ch. E.; May, W. H., y Miron, M. S. (1975), Cross Cultural Universals of Affective Meaning, Urbana, University of Illinois Press.

Oyama, S. (1992), «Is Phylogeny recapitualting Ontogeny?», en VArela, F. J., y DupuY, J. P. (eds.), Understanding origins, Dordrecht, Kluwer, pp. 227-232.

Papataxiarchis, E. (1994), «Émotions et stratégies d'autonomie en Grèce égéenne», Terrain, núm. 22, marzo, pp. 5-20.

Peixoto, C. (1995), «Les modes d'appellation dans les lieux publics. Une comparaison entre la France et le Bresil», Ethnologie francaise, núm. 4, pp. 559-568.

Perret, D. I. P., et al. (1995), «When is a face not a face? Correlations between perception and single neurons», en GRE-
GOry, R., y Harris, J. (ed.), The Artful Eye, Oxford, Oxford University Press, pp. 95-124.

Perret, D. I. P.; Rolls, E. T., y CaAn, W. (1982), «Visual neurones responsive to faces in monkey temporal cortex», Experimental Brain Research, Berlín, núm. 47, pp. 329-342.

Pinol-Douriez, M. (1987), «Affect et Processus de symbolisation. Les interrelations precoces», en Fondation Archives Jean Piaget, Symbolisme et connaissan$c e$, Genève, Université de Genève, pp. 169-187.

Premack, (1980), "Characteristics of an upgraded mind», en Bericht uber den 32. Kongress der Deutschen Gesellschaft fur Psychologie in Zurich, W. Michaelis, vol. 1, pp. 49-70, cit. en Lock \& Colombo, 1996 .

- (1984), «Pedagogy and Aesthetics as sources of culture», en Gazzaniga, M. S. (ed.), Handbook of cognitive neuroscien$c e$, Londres, Plenum Press.

Putnam, H. (1981), Reason, Truth and History, Cambridge, CUP.

- (1999), The threefold cord: Mind, Body, and World, Nueva York, Columbia University Press.

Ramírez Goicoechea, E. (1991), De jóvenes y sus identidades. SocioAntropología de la Etnicidad en Euskadi, CIS/S. XXI, Madrid, 1991.

- (1998), Basic Sociality and Othernes. Building Blockd for a New Paradigm in the Social Sciences. XIVth World Congress of Sociology. Montréal, Canada. (RC33 'Researching Neglected Topics).

Reite, M., y Short, R. (1981), "Attachmente, loss and depression», Journal of Child Psychology and Psychiatry, núm. 2, pp. 141-170.

RoBertson, A. F. (1996), «The development of meaning: Ontogeny and culture», Man (NS), vol. 2, núm. 4, pp. 591-610.

Rolls, E. T. (1994), «A theory of emotion and consciousness, and its application to understanding the neural basis of 
emotion», en Gazzinaga, M. (ed.), The Cognitive Neurosciences, Harvard, Mass., MIT Press.

Rosaldo, M. (1980), Knowledge and passion: Ilongot notions of self and social life, Cambridge, CUP.

- (1983), «The Shame of Headhunters and the Autonomy of Self», Ethos, 11:3, pp. 135-151.

- (1984), «Toward and anthropology of self and feeling», en SHweder, R., y LEVIne, R. (eds.), Culture theory: essays on mind, self and emotion, Cambridge, CUP, pp. 137-157.

Rosenberg, D. V. (1990), «Language in the discourse of emotions», en LuTz, C. A., y Abu-Lughod, L. (dirs.), Language and the Politics of Emotion, Cambridge, CUP.

Ross, E. D., y Mesulam, M. M. (1979), «Dominant language functions of the right hemisphere: prosody and emotional gesturing», Archives of Neurology, núm. 36, pp. 144-148.

Sagi, A.; LAmb, M. E.; Lewkowicz, K.; Shoham, R.; Dvir, R., y Estes, D. (1985), «Security of infant-mother, -father, and -metapelet attachments among kibbutz-reared israeli children», en BRETHERTON, I., y Waters, E., Growing points in attachment theory, Monographs for the Society for Research in Child Development, serial núm. 209, vol. 50 (1\&2), pp. 257-275.

SCHAFFER, H. R. (1992), «Joint Involvement Episodes as Context for Development», en H. McGurk, Childhood Social Development, Hove, NJ, Earlbaum, pp. 99-129.*

Schank, R. G., y Abelson, R. (1977), Scripts, plans, goals and understanding, Hillsdale, NJ, Erlbaum.

SCHEFF, Th. (1990), «Sozialization of emotions: pride and shame as causal agents», en Kemper, T. D. (ed.), Research Agendas in the Sociology of Emotions, Nueva York, State University of New York Press.
SchiefFelin, E. L. (1983), «Anger and Shame in the Tropical Forest: On Affect as a Cultural System in Papua New Guinea», Ethos, núm. 11, pp. 181-191.

SeArle, J. R. (1990), «Is the Brain's Mind a Computer Program?», Scientific American, 262 (1), pp. 20-25.

Seymour, S. (1983), «Household Structure and Status and Expressions of Affect in India», Ethos, 11:4, pp. 263-277.

Shilling, C., y Mellor, P. (1996), «Embodiment, structuration theory and modernity: mind/body dualism and the repression of sensuality», Body \& Society, 2 (4), pp. 1-15.

Shore, B. (1996), Culture in Mind. Cognition, culture and the problem of meaning, Oxford, Oxford University Press.

Shweder, R. A. (1994), "“You're not sick, You're Just in Love": Emotion as an Interpretative system», en EkMAN, P., y Davidson, R. J. (eds.), The nature of Emotion: Fundamental Questions, Nueva York, Oxford University Press, pp. 32-44.

Shweder, R. A., y LeVIne, R. (eds.) (1984), Culture Theory: Essays on mind, self and emotion, Cambridge, CUP.

Solomon, R. C. (1984), «Getting Angry. The Jamesian Theory of Emotion in Anthropology», en SHweder, R., y LEVINE (eds.), Culture Theory. Essays on Mind, Self, and Emotion, Cambridge, CUP, pp. 238-254.

SPERBER, D. (1994), «The modularity of thought and the epidemiology of representations», en Hirschfeld, L. A., y Gelman, S. A. (eds.), Mapping the mind. Domain specificity in cognition and culture, Cambridge, Cambridge University Press, pp. 39-67.

Sperber, D., y Wilson, D. (1986), Relevance. Communication and Cognition, Oxford, Basil Blackwell.

Stephan, F. K.; Berkley, K. J., y Moss, R. L. (1981), «Efferent connections of the rat suprachiasmic nucleus», Neuroscience, núm. 6, pp. 2625-2641. 
Stocking, G. W. (1982) (1968), Race, culture and evolution: essays in the History of Anthropology, Chicago, University Press.

Tomkins, S. S. (1981), «The quest for Pimary Motives: biography and autobiography of an Idea», Journal of Personality and socil Psychology, 41,2, pp. 306-329.

Toren, C. (1993), «Making History: the Significance of Childhood Cognition for a Comparative Anthropology of Mind», en Man (NS), núm. 28, pp. 461-478.

Trevarthen, C. (1980), «The foundations of intersubjectivity: development of interpersonal and co-operative understanding in infants», en OLson, D. (ed.), The social foundations of language: essays in honor of J.S. Bruner, Nueva York, Norton, pp. 316-342.

- (1986), «Development of intersubjective motor control», en WADE, M., y Whiting, H. T. A. (eds.), Motor development in children, The Hague, Martinus Nijhoff.

TuCKer, D. M. (1981), «Lateral brain function, emotion, and conceptualization», Psychological Bulletin, núm. 89, pp. 19-46.

TuRner, V. (1977), The ritual process: structure and antistructure, Ithaca, Cornell University Press.

- (1980) (1967), La selva de los símbolos, Madrid, S. XXI.

VAndamme, F. (1988), «Emotion, cognition, intelligence and meaning in an Artificial Intelligence perspective», en Carvallo, M. E. (ed.), Nature, Cognition and System I, Dordrecht, Kluwer Academic Publishers, pp. 61-75.

Varela, J. F.; Thompson, E., y Rosch, E., et al. (1991), The embodied mind: cognition scienceand human experience, Cambridge, Mass., MIT Press.

Wentworth, W. M., y Yardley, D. (1994), «Deep sociality: a bioevolutionary pers- pective on the sociology of emotions», en Wentworth, W. M., y Ryan, J. (eds.), Social Perspectives on Emotion, Greenwitch, CT: JAI Press.

Whitehouse, H. (1996), «Rites of Terror: Emotion, Metaphor and Memory in Melanesian Initiation Cults», Journal of the Royal Anthropological Institute (N.S.), núm. 2, pp. 703-715.

Whiten, A. (1991), «The emergence of metarepresentation in human ontogeny and primate phylogeny», en Whiten, A. (ed.), Natural theories of mind. Evolution, development and simultion in everyday mind reading, Oxford, Basil Blackwell.

- Byrne, R. W. (1988), «The manipulation of attention in primate tactical deception», en Whiten, A., y Byrne, R. W., Machiavellian intelligence: Social expertise and evolution of intellect in monkeys, apes and humans, Oxford, Oxford University Press.

WierzbickA, A. (1986), «Human Emotions: Universal or culture specific», American Anthropologist, núm. 88, pp. 584-594.

- 1994, «Cognitive domains and the structure of the lexicon: The case of emotions», en HirschFeld, L. A., y GeLMAN, S. A. (eds.), Mapping the mind. Domain specificity in Cognition and Culture, Cambridge, CUP, pp. 431-452.

Williams, S. (2001), Emotion and Social Theory, Londres, SAGE.

Williams, S., y Bendelow, G. (1998), «In search of the missing body? Pain, suffering and the (post)modern condition», en Scambler, G., y Higgs, P. (eds.), Modernity, Medicine and Healt, Londres, Routledge.

Zajonc, R. B. (1984), «On the Primacy of Affect», American Psychologist, 39, 2, pp. 117-123. 


\section{NOTAS}

1 Aquí, como en muchas aproximaciones biologicistas, se confunde lo «innato» con lo «involuntario». El aprendizaje y el hábito bien pueden producir patrones automáticos de respuesta.

${ }^{2}$ Esta paradoja entre la defensa de la unidad psíquica del hombre/mujer y la diversidad cultural ha sido arrastrada por prácticamente toda la antropología desde Lévi-Bruhl, Lévi-Strauss, hasta Geertz (Shore, 1996).

3 Ángel Díaz de Rada, Comunicación personal.

${ }^{4}$ Ginsburg y Harrington (1996) hasta cuestionan esta generalidad incluso para las emociones más primarias. Por su parte, J. R. Averill no encuentra patrones comunes para las emociones «intelectuales» como la esperanza («hope») (Averill, 1996a). Esto no quiere decir, en nuestra opinión, que no haya actividad corporalizada de alguna manera, sino que el cuerpo es capaz de «olvidarse» de sí mismo, lo que ha sido confundido por algunos con una facultad a-corporalizada de la mente (cf. Leder, 1990).

5 La convencionalidad, es decir, la creación de un significado social compartido, se ha confundido también con la inmotivación en lingüística.

6 La noción de «image schematta» de Johnson (1987), como una serie limitada de esquemas experienciales corporales que sirven como bases conceptuales para otros dominios de la experiencia, entre ellos la cognición, tendría utilidad heurística aquí. A partir de estos esquemas se elaborarían diversas metáforas explicativas. Véase también la teoría «integrada» de metáforas primarias elaborada en Lakoff y Jonson (1999).

${ }^{7} \mathrm{Su}$ volumen crece hasta el primer año de edad, continuando la mielización del córtex a lo largo de los primeros años (Gibson, 1996). Incluso hay evidencias neurológicas del «reparto» del complejo proceso emocional entre los dos hemisferios durante el primer año de vida. Cf. Davidson, 1984

${ }^{8}$ Un sistema autopoiético, al fin y al cabo. Cf. Maturana y Varela, 1980.

Más que de tratar de asignar un cierto nivel de capacidad a una especie en particular, habría que tener en cuenta las circunstancias (una ecología) en que estas capacidades aparecen. Cf. Lock y Colombo, 1996; Bateson, 1973.

${ }^{10}$ En el mismo sentido de que Premack cita para los chimpancés de laboratorio, en donde las tecnologías y condiciones experimentales de laboratorio (que, sin embargo, para los humanos constituyen su situación «natural» de existencia) trabajan «ampliando» («upgrading») las capacidades cognitivas de estos primates (Premack, 1980, cit. en Lock y Colombo, 1996).

${ }_{11}$ Los análisis psicológicos basados en la «personalidad» destacarían las características modales individuales como rasgos psíquicos propios de cada uno, independientes no sólo de factores interactivos, sino también, por supuesto, de variables socioculturales.
${ }^{12}$ El feedback que se produce cuando se simula la expresión de una emoción en la cara sobre la activación y control de dicha emoción en el cerebro tiene un refrendo empírico neurofisiológico por medio de la actividad del nervio trigémino. W. James reconocía la importancia de la retroalimentación efectuada desde los músculos a los centros de actividad neurológica. Claro está que investigadores como P. Ekman, contra quienes van dirigidas estas críticas precisamente, lo ponen en duda. Comunicación pública. Sixth International Colloquium on Cognitive Science, 1999. San Sebastián. En todo caso, esta capacidad de sentir emoción a partir también de la expresión de la misma parece tener un papel importante en el establecimiento de la empatía que hemos citado. Cf. infra.

${ }^{13}$ Lo que ocurre es que esta perspectiva más que integradora suele ser cognitivista. Cf. Greenberg y Safran, 1987.

${ }^{14} \mathrm{La}$ conocida teoría de W. James señala que la emoción sería precisamente, esta toma de conciencia, pero de cambios corporales que se producen como reacción a determinadas situaciones.

${ }^{15}$ Para Sperber y Wilson (1986), la «relevancia» de algo se refiere a la ampliación de nuestro acervo cognitivo en el sentido representacional, lejos de incluir ningún aspecto emocional. Implica al sujeto como ente cognoscente, pero no como sujeto experiencial.

${ }^{16}$ Sobre género y dualismo mente/cuerpo véase, entre otros, Williams y Bendelow (1988) y Lupton (1998)

17 Maturana y Varela, 1992, mencionan el caso de ovejas a las que su madre no ha lamido y acariciado en su primera infancia y que muestran deficiencias posteriores a la hora del juego con otros de su mism especie. Harlow y Harlow (1996) citan la disminución de la exploración del entorno y la agresividad injustificada en monos separados tempranamente de sus madres. Los cambios fisiológicos operados en éstos ante la separación de sus madres son descritos en Reite y Short (1981), quienes también comprobaron la pérdida de interés por el entorno.

${ }^{18}$ Sobre esta apasionante cuestión no podemos extendernos por el momento. Véase Gómez y Núñez, 1998. En Byrne y Whitten (1995) también se parte de una inteligencia diferencial para personas, grupos, relaciones sociales, y objetos naturales y artefactos, como en la mayoría de los autores que sostienen las teorías de especificidad de dominio, pero la visión es absolutamente opuesta: aquélla está guiada por el interés y la racionalidad (una orientación «maquiavélica») no por componentes afectivos.

${ }_{19}$ En referencia concreta al campo de las emociones, Williams (2001) también cita el existencialismo de Sartre.

${ }^{20}$ Nuestras principales referencias aquí son, sobre todo, las de F. Varela et al., 1991; M. Johnson (1987); G. Lakoff y Johnson (1999); C. Toren (1993); Csordas (1994); Haraway (1990, 1991). 